\title{
FREQUÊNCIA DE EXACERBAÇÃO EM PACIENTES PORTADORES DE DPOC SUBMETIDOS A UM PROGRAMA DE REABILITAÇÃO PULMONAR
}

\author{
FREQUENCY OF EXACERBATIONS IN PATIENTS \\ DIAGNOSED WITH COPD SUBMITTED IN \\ PULMONARY REHABILITATION PROGRAM
}

\author{
Alice Pereira Freitas' ${ }^{1}$ Betina Brixner', Cristiane Carla Dressler Garske', \\ Andréa Lúcia Gonçalves da Silva², Dulciane Nunes Paiva ${ }^{3}$, \\ Dannuey Machado Cardoso ${ }^{4}$, Lisiane Lisboa Carvalho ${ }^{5}$
}

\begin{abstract}
RESUMO
O objetivo deste estudo foi avaliar a frequência de exacerbação em pacientes diagnosticados com Doença Pulmonar Obstrutiva Crônica (DPOC) submetidos em Programa de Reabilitação Pulmonar (PRP) em hospital de ensino no interior do Rio Grande do Sul. Estudo transversal com 32 pacientes. A coleta de dados ocorreu em outubro de 2015, através do sistema computadorizado do hospital e banco de dados do PRP. A amostra foi estratificada em grupos, conforme o tempo de permanência no PRP: menos de dois meses (G1), de dois a 12 meses (G2) e mais de 12 meses (G3). Verificou-se que $59,4 \%$ eram do sexo masculino com idade média de 63,5 anos $( \pm 6,9)$. No $\mathrm{G} 1,50 \%$ dos pacientes exacerbaram, no $\mathrm{G} 2,65 \%$ e no $\mathrm{G} 3,37,5 \%$. Os resultados demonstraram que portadores de DPOC que frequentaram o PRP por tempo superior a 12 meses apresentaram menor frequência e menor intensidade de exacerbação da doença.
\end{abstract}

Descritores: Reabilitação; Doença Pulmonar Obstrutiva Crônica; Exacerbação dos Sintomas.

\begin{abstract}
The aim of this study was to evaluate the frequency of exacerbations in patients diagnosed with Chronic Obstructive Pulmonary Disease (COPD) submitted in Pulmonary Rehabilitation Program (PRP) in teaching hospital in the Rio Grande do Sul. Cross-sectional study with 32 patients. Data collection took place in October 2015, through the computerized system of the hospital and the PRP database. The sample was divided into groups according to the residence time in the PRP: less than two months (G1) of two to 12 months (G2) and more than 12 months (G3). It was found that $59.4 \%$ were male with a mean age of 63.5 years $( \pm 6.9)$. In $\mathrm{G} 1,50 \%$ of exacerbated patients, $\mathrm{G} 2, \mathrm{G} 3$ and $65 \%, 37.5 \%$. The results showed that patients with COPD who attended the PRP for longer than 12 months had a lower frequency and lower intensity of disease exacerbation.
\end{abstract}

${ }^{1}$ Graduada em Farmácia pela Universidade de Santa Cruz do Sul (UNISC), Santa Cruz do Sul, RS, Brasil.

${ }^{2}$ Doutora em Biologia Celular e Molecular pela Universidade Federal do Rio Grande do Sul (UFRGS), Porto Alegre, RS, Brasil.

${ }^{3}$ Doutora em Ciências Pneumológicas pela Universidade Federal do Rio Grande do Sul (UFRGS), Porto Alegre, RS, Brasil.

${ }^{4}$ Mestre em Ciências Médicas pela Universidade Federal do Rio Grande do Sul (UFRGS), Porto Alegre, RS, Brasil.

${ }^{5}$ Mestre em Promoção da Saúde pela Universidade de Santa Cruz do Sul (UNISC), Santa Cruz do Sul, RS, Brasil. 


\section{Introdução}

As doenças respiratórias crônicas como asma, rinite alérgica e a DPOC representam um dos maiores problemas de saúde mundial ${ }^{1}$. De acordo com a Global Initiative for Chronic Obstructive Lung Disease ${ }^{2}$ a DPOC caracteriza-se por obstrução persistente e geralmente progressiva que acomete as vias aéreas e os pulmões. É uma doença que não possui cura, mas com 0 tratamento adequado seu progresso pode ser retardado ${ }^{3}$. Outras comorbidades, bem como exacerbações podem contribuir para a gravidade dos pacientes. As exacerbações são recorrentes, manifestando-se através de alterações clínicas do paciente e um aumento dos sintomas respiratórios 4 .

As exacerbações da DPOC se apresentam como um fator de risco para a piora do paciente, e consequentemente um aumento da morbimortalidade ${ }^{5}$. Muitas vezes ocorrem desencadeadas por infecções, bacterianas ou virais, fatores ambientais, condições como pneumonia, tromboembolismo e insuficiência cardíaca aguda, ou ainda por fatores desconhecidos. $O$ aumento da dispneia em pacientes exacerbados ocorre devido a hiperinsuflação e aprisionamento de gás gerando a redução do fluxo respiratório ${ }^{3}$.

Conforme estudo ${ }^{4}$, as exacerbações podem ser leves, moderadas ou graves. As exacerbações leves podem ser descritas como aquelas em que o paciente tem um aumento da necessidade de medicação, podendo ser tratadas em domicílio, fato pelo qual as mesmas não foram consideradas nesse estudo. Nas exacerbações moderadas, o paciente tem aumento da necessidade de medicação e percebe a necessidade de auxílio médico adicional o que o faz antecipar uma consulta ou recorrer a um serviço de emergência. Na exacerbação grave, o paciente e/ou seu cuidador ao reconhecer uma piora significativa e/ou rápida da condição clínica procura atendimento sendo conduzido a internação hospitalar.

ADPOC afeta 210 milhões de pessoas, sendo considerada a quarta causa de mortalidade em todo o mundo. De acordo com a Organização Mundial da Saúde, em 2012, a DPOC foi responsável por 6\% dos óbitos no mundo, o que equivale ao acometimento de cerca de três milhões de pessoas, devendo ser ressaltado que cerca de $90 \%$ dos óbitos ocorreu em pacientes de baixa e média renda, afetando homens e mulheres de modo similar ${ }^{3}$.

No Brasil a DPOC encontra-se entre a quinta e sexta principal causa de morte e estima-se que 7,5 milhões de pessoas sejam portadoras de DPOC. No ano de 2008 foram realizadas 170 mil admissões no Sistema Único de Saúde (SUS) de pacientes com DPOC com a permanência média de internação de seis dias¹.

$\mathrm{O}$ crescente aumento da mortalidade causada pela DPOC impulsionou a busca de alternativas de tratamento farmacológico e não farmacológico. A reabilitação pulmonar se caracteriza por um tratamento não farmacológico capaz de reduzir os sintomas da doença, aumentar a qualidade de vida e reduzir custos de despesas relacionadas com a saúde, revertendo ou estabilizando os efeitos da doença ${ }^{2}$. Dessa forma, o presente estudo objetivou avaliar a frequência de exacerbação por DPOC em sujeitos submetidos a um PRP em um hospital de ensino no interior do Rio Grande do Sul.

\section{Metodologia}

Trata-se de um estudo transversal, realizado em um PRP, situado juntamente a um hospital de ensino no interior do Rio Grande do Sul - Brasil, com coleta de dados em outubro de 2015.

Dentre os critérios de inclusão estão: pacientes de ambos os sexos, idade acima de 18 anos e participantes do PPSUS. Como critérios de exclusão, os pacientes que possuíam convênios privados de saúde e os pacientes que não completaram as avaliações para iniciar as atividades no PRP.

Foram selecionados 38 pacientes para as atividades no Programa de Pesquisa para o SUS (PPSUS), que visou acompanhar os pacientes previamente diagnosticados com DPOC por dois anos (setembro de 2013 a setembro de 2015). Cinco pacientes foram excluídos, pois não havia completado os testes necessários para iniciar o acompanhamento no programa e um foi excluído por apresentar convênio privado de saúde. Resultando em amostra final de 32 pacientes.

As informações foram coletadas através do sistema informatizado $\mathrm{MV}_{200{ }^{\circledR}}$ e prontuários médicos, buscando identificar a causa e a frequência da exacerbação, e os dados dos pacientes e outras variáveis (sexo, idade, hábito tabagista, Volume Expiratório Forçado no primeiro segundo (VEF1, VEF1 predito) e estadiamento da doença) foram coletadas através de banco de dados do PRP. Comparou-se, também, a frequência da exacerbação com o tempo de permanência no PRP.

Os pacientes avaliados foram estratificados em três grupos: aqueles que não completaram dois meses das atividades (G1); aqueles que realizaram as atividades entre dois a doze meses (G2); e aqueles que realizaram as atividades por um período maior de doze meses (G3). 
A exacerbação foi classificada quanto aos seus estádios de gravidade como moderada quando necessitou de atendimento ambulatorial e grave quando necessitou internação hospitalar, não sendo levado em consideração o estádio leve, pois este se trata de alterações que não necessitam atendimento, podendo ser resolvidas no próprio domicílio do paciente.

Utilizou-se 0 software Microsoft Excel $2007^{\circledR}$ para registrar os dados originais e para a análise exploratória, posteriormente gerando tabelas e gráficos.

Este estudo foi isento de termo de consentimento livre e esclarecido, devido ao anonimato dos pacientes e a isenção de intervenções, fazendo parte de uma pesquisa maior, que inclui desfechos não mencionados neste artigo. 0 projeto de pesquisa foi aprovado pelo Comitê de Ética em Pesquisa da Universidade de Santa Cruz do Sul sob o número 435.093/2013.

\section{Resultados}

Dos 32 pacientes participantes do estudo, a idade média encontrada foi de 63,5 $\pm 6,9$ anos, sendo a idade mínima e máxima, respectivamente, 50 e 79 anos. Referente ao sexo, 19 (59,4\%) era paciente do sexo masculino e 13 (40,6\%) do sexo feminino.

Dos indivíduos participantes do estudo, 2 (6,3\%) continuam com o hábito tabagista e $30(93,7 \%)$ pararam de fumar; 8 (25\%) pacientes eram de estadiamento moderado, 14 (43,7\%) eram grave e 10 (31,3\%) muito grave; o VEF 1 predito variou de 12 a $72 \%$, com média de $39,4 \% \pm 15,7 \%$, o VEF 1 de 0,25 a 2,12, com média de 1,03 $\pm 0,48$.

Os resultados encontrados com a frequência de exacerbações estão descritos na Tabela 1, onde exacerbaram $50 \%$ dos quatro pacientes pertencentes ao $\mathrm{G} 1,65 \%$ dos vinte pacientes do $\mathrm{G} 2$ e $37,5 \%$ dos oito pacientes do $\mathrm{G} 3$.

Tabela 1 - Frequência de exacerbações em pacientes diagnosticados com DPOC durante o período da pesquisa no PRP do hospital de ensino do interior do Rio Grande do Sul - Brasil, conforme estratificações dos grupos de permanência no PRP

\begin{tabular}{|c|c|c|c|c|}
\hline & G1 & $\mathbf{G 2}$ & G3 & Total \\
\hline Sem exacerbação & $2(50 \%)$ & 7 (35\%) & $5(62,5 \%)$ & $14(43,7 \%)$ \\
\hline \multirow[t]{5}{*}{ Exacerbação } & $1(25 \%)-1^{*}$ & $7(35 \%)-1^{*}$ & $2(25 \%)-2^{*}$ & \multirow{5}{*}{$18(56,3 \%)$} \\
\hline & $1(25 \%)-4^{*}$ & $2(10 \%)-6^{*} / 7^{* *}$ & $1(12,5 \%)-1^{*}$ & \\
\hline & & $2(10 \%)-3^{*} / 7^{* *}$ & & \\
\hline & & $1(5 \%)-14^{*} / 2^{* *}$ & & \\
\hline & & $1(5 \%)-2^{*}$ & & \\
\hline Total & $4(100 \%)$ & 20 (100\%) & 8 (100\%) & 32 (100\%) \\
\hline
\end{tabular}

*Número de exacerbação moderada.

**Paciente que, além de exacerbação moderada, apresentou exacerbação grave.

Ao longo dos dois anos de extensão do projeto, 19 pacientes desistiram do programa, sendo assim, 11 pacientes continuaram em acompanhamento das atividades e 2 foram a óbito.

\section{Discussão}

Um dos principais achados deste estudo em portadores de DPOC acompanhados durante dois anos em PRP é que os pacientes que frequentaram por mais de doze meses o PRP apresentaram menores frequências e menor intensidade de exacerbações.

Neste estudo, a prevalência de pacientes com DPOC foi do sexo masculino $(59,4 \%)$ e a idade média encontrada entre os participantes foi de $63,5 \pm 6,9$ anos. Estes resultados encontrados são próximos ao de um estudo ${ }^{6}$ realizado 
no Hospital Masih-Daneshvari, no Irã, com 66 pacientes portadores de DPOC realizando atividades de reabilitação pulmonar, onde a média de idade foi de 56,6 $\pm 8,8$ anos e $71,2 \%$ eram pertencentes ao sexo masculino.

No estudo, dos 32 pacientes, somente 2(6,3\%) continuaram com o hábito tabagista ao longo do PRP, e 30 (93,7\%) pararam de fumar, estudos demonstram que frequentando o PRP, os pacientes recebem estímulo da equipe para cessar o hábito e também dos próprios ex-fumantes participantes do PRP7 . Outro estudo descreve que aqueles pacientes com função pulmonar mais debilitada ou que apresentam exacerbações frequentes tendem a deixar de fumar, diferentemente daqueles pacientes com menos severidade da doença ${ }^{8}$.

Em relação aos valores médios encontrados para VEF1 $(1,03)$ e VEF1 predito $(39,7)$, obtidos através dos testes de espirometria que os pacientes realizaram, é possível verificar a gravidade da obstrução do fluxo aéreo. Estes resultados demonstram que a população atendida no PRP possui uma capacidade respiratória diminuída e, além disso, segundo os critérios de GOLD, a relação de VEF1/Capacidade de Volume Forçado (CVF) $\leq 70 \%$ do valor previsto já é considerado como um diagnóstico de DPOC, onde os estágios da DPOC são baseados no VEF1 pós-broncodilatador².

A reabilitação pulmonar é uma importante estratégia para mudança de comportamentos em saúde, o qual é vital para melhorar e manter os benefícios de intervenções em pacientes com DPOC. Dentre os benefícios que a reabilitação pulmonar traz aos pacientes, citam-se: melhora da sensação de dispneia, aumento da qualidade de vida, melhor capacidade de realizar exercícios, diminuição de internações hospitalares e dos episódios de depressão e ansiedade ${ }^{9}$. Estas informações condizem com o resultado encontrado no presente estudo, uma vez que os pacientes que permaneceram por um período mais longo no programa (G3), realizando as atividades de reabilitação pulmonar, apresentaram menos episódios de exacerbações (37,5\%) quando comparado ao G2 (65\%); sendo que no G3 a intensidade da exacerbação também foi menor, onde nenhum paciente apresentou exacerbação grave.

Os pacientes com DPOC, participantes de um PRP apresentam redução na frequência de exacerbação e hospitalização, o que influencia positivamente na progressão da doença e na redução dos custos com saúde ${ }^{10}$. Sabe-se que o aumento do número de exacerbações está diretamente relacionado com a gravidade da DPOC e com diminuição da função pulmonar; dessa forma, a exacerbação deve ser reconhecida e tratada rapidamente de forma que o paciente tenha a menor perda funcional possível ${ }^{11}$. Para o tratamento ideal da DPOC é necessária persistência nas mudanças de hábitos e estilo de vida, e a participação em PRP promove melhorias na qualidade de vida do paciente a curto e longo prazo ${ }^{12}$.

Neste estudo, consegue-se observar a melhoria da qualidade de vida desses pacientes submetidos a PRP, confirmando que quanto mais tempo permanecer no programa realizando as atividades físicas, menos exacerbações irão apresentar ao longo da vida. Como os pacientes do G1 permaneceram por menos de dois meses, não houve seguimento das atividades no PRP sendo acompanhados somente quando retornaram ao hospital para atendimento de suas exacerbações. Os pacientes do G2, além de exacerbações moderadas, apresentaram também exacerbações graves, o que pode sugerir a falta de adesão do tratamento farmacológico, pois, apesar de não ter sido um dado levantado nos pacientes em questão, estudos ${ }^{13}$ destacam a importância dos medicamentos na melhora da função pulmonar dos pacientes com DPOC. Já os do G3, foram os que menos apresentaram exacerbações moderadas durante o estudo e nenhum paciente deste grupo necessitou de internação hospitalar (exacerbação grave).

Este estudo reforça que são necessárias medidas que possam minimizar a piora dos sintomas e o trabalho deve ser realizado por uma equipe multiprofissional, o que permitirá a evolução de outros aspectos nos pacientes portadores de DPOC ${ }^{14}$.

\section{Considerações Finais}

Com o presente estudo, concluiu-se que pacientes que frequentaram por mais de doze meses o PRP apresentaram menor frequência e intensidade de exacerbação. Desta forma, as evidências sugerem que o programa de reabilitação, em regime ambulatorial, com equipe multiprofissional, citado neste estudo, contribui para este resultado.

\section{Referências}

1. Brasil. Ministério da Saúde. Secretaria de Atenção à Saúde. Departamento de Atenção Básica. Doenças respiratórias crônicas/Ministério da Saúde, Secretaria de Atenção à Saúde, Departamento de Atenção Básica. - Brasília: Ministério da Saúde, 2010. 160 p.: il. - (Série A. Normas e Manuais Técnicos) (Cadernos de Atenção Básica, n. 25). 
2. GOLD - Global Initiative for Chronic Obstructive Lung Disease. Global strategy for the diagnosis, management, and prevention of chronic obstructive pulmonary disease. Updated 2015. National Institutes of Health and National Heart, Lung and Blood Institute. 3. World Health Organization.Programmes and Projects.Chronic respiratory diseases - Chronic Obstructive Pulmonary Disease (COPD).Fact Sheet, 315. January, 2015.

4. Cardoso AP. Exacerbação da DPOC. Pulmão. 2013;22(2):60-64.

5. Galhardo, M. Corticoterapiana exacerbação de doença pulmonar obstrutiva crônica - qual a melhor opção: duração curta ou convencional? Ver Port Med Geral Fam. 2013;29(5):344-346.

6. KhoshkeshtS, Zakerimoghadam M, Ghiyasvandian S, KazemnejadA, Hashemian M. The effect of home-based pulmonary rehabilitation on self-efficacy in chronic obstructive pulmonary disease patient. J Pak MedAssoc. 2015;65(10):1041-1046. 7. Squassoni SD, Lapa MS, Fiss E. Efeitos da reabilitação pulmonar em pacientes fumantes e ex-fumantes com doença pulmonar obstrutiva crônica. Arq Bras Ciên Saúde. 2011;36(1):18-23.

8. Niewoehner DE, Lokhngina Y, Rice K, Kuschner WG, Sharafkhaneh A, Sarosi GA et al. Risk indexes for exacerbations and hospitalizations due to COPD. Chest. 2007;131(1):20-28.

9. Spruit MA, Singh SJ, Garvey C, ZuWallack R, Nici L, Rochester C. An official American Thoracic Society/European Respiratory Society statement: key concepts and advances in pulmonary rehabilitation. AmJ RespiCrit Care Med. 2013;189(12):1570.

10. Ranst DV, Stoop WA, Meijer JW, Otten HJ, Port IGV. Reduction of exacerbation frequency in patients with COPD after participation in a comprehensive pulmonary rehabilitation program.International. Journal of COPD. 2014;9(1);1059-1067.

11. Costa CH, Rufino, R. Tratamento da doença pulmonar obstrutiva crônica. Rev HUPE. 2013;12(2):71-77.

12. Ochmann U, Rudolf AJ, Dennis N. Long-term Efficacy of Pulmonary Rehabilitation: a state of the art review. Jou Cardiopul Rehab Prev. 2012;32(1):117-126.

13. Wehrmeister FC, Knorst M, Jardim JR, Macedo EC, Noal RB, Martinez-Mesa J et al. Pulmonary rehabilitation programs for patients with COPD. J Bras Pneumol. 2011;37(4):544-555.

14. Campos AA, Cabrera R, Osvaldo CR. Rehabilitación respiratoria en pacientes

EPOC: experiencia en Atención Primaria de Salud. Rev Chil Enferm Respir.2015;31:77-85.

\author{
Alice Pereira Freitas \\ Endereço para correspondência - Rua: São João, $n^{\circ} 66$, \\ Bairro: Centro, CEP: 95820000 , General Câmara, RS, Brasil. \\ E-mail: alicefreit@hotmail.com \\ Lattes: http://lattes.cnpq.br/9653671435069536 \\ Betina Brixner - betinabrixner@yahoo.com.br \\ Cristiane Carla Dressler Garske - crisdressler2005@yahoo.com.br \\ Andréa Lúcia Gonçalves da Silva - andreag@unisc.br \\ Dulciane Nunes Paiva - dulciane@unisc.br \\ Dannuey Machado Cardoso - dannueyc@unisc.br \\ Lisiane Lisboa Carvalho - lisianecarvalho@unisc.br
}

\title{
Enviado em 07 de outubro de 2016. Aceito em 15 de março de 2017.
}

